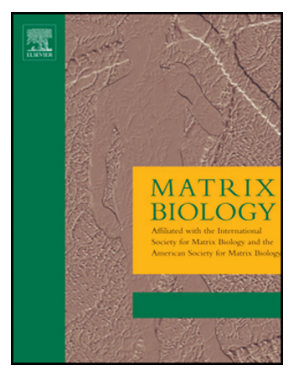

\title{
Biophysical analysis of a lethal laminin alpha-1 mutation reveals altered self-interaction
}

\author{
Trushar R. Patel $^{a, b \dagger}$, Denise Nikodemus ${ }^{c, \dagger}$, Tabot M.D. Besong ${ }^{d, e}$, \\ Raphael Reuten ${ }^{c}$, Markus Meier ${ }^{a}$, Stephen E. Harding ${ }^{d}$, Donald J. Winzor ${ }^{f}$, \\ Manuel Koch ${ }^{\mathrm{b}}$ and Jörg Stetefeld ${ }^{\mathrm{a}}$,
}

\begin{abstract}
a - Department of Chemistry, University of Manitoba, 144 Dysart Road, Winnipeg, Manitoba R3T 2N2, Canada
b - School of Biosciences, University of Birmingham, Edgbaston, Birmingham B15 2TT, United Kingdom

c - Institute for Dental Research and Oral Musculoskeletal Biology and Center for Biochemistry, Medical Faculty, University of Cologne, Cologne 50931, Germany

$\boldsymbol{d}$ - National Center for Macromolecular Hydrodynamics, University of Nottingham, Sutton Bonington LE12 5RD, United Kingdom e - Functional Nanomaterials Lab, King Abdullah University of Science and Technology (KAUST), Thuwal 23955-6900, Saudi Arabia $\boldsymbol{f}$ - School of Chemistry and Molecular Biosciences, University of Queensland, Brisbane, Queensland 4072, Australia
\end{abstract}

Correspondence to Trushar R. Patel and Jörg Stetefeld: T. R. Patel, University of Birmingham, Edgbaston, Birmingham, B15 2TT, United Kingdom. J. Stetefeld, University of Manitoba, 144 Dysart Road, Winnipeg, Manitoba R3T 2N2, Canada. t.r.patel@bham.ac.uk; jorg.stetefeld@ad.umanitoba.ca http://dx.doi.org/10.1016/j.matbio.2015.06.005

Edited by R. lozzo

\begin{abstract}
Laminins are key basement membrane molecules that influence several biological activities and are linked to a number of diseases. They are secreted as heterotrimeric proteins consisting of one $\alpha$, one $\beta$, and one $y$ chain, followed by their assembly into a polymer-like sheet at the basement membrane. Using sedimentation velocity, dynamic light scattering, and surface plasmon resonance experiments, we studied self-association of three laminin (LM) N-terminal fragments $\alpha-1$ (hLM $\alpha-1 \mathrm{~N}), \alpha-5$ (hLM $\alpha-5 \mathrm{~N})$ and $\beta-3(\mathrm{hLM} \beta-3 \mathrm{~N}$ ) originating from the short arms of the human laminin $\alpha \beta y$ heterotrimer. Corresponding studies of the hLM $\alpha-1 \mathrm{~N} C 49 \mathrm{~S}$ mutant, equivalent to the larval lethal C56S mutant in zebrafish, have shown that this mutation causes enhanced self-association behavior, an observation that provides a plausible explanation for the inability of laminin bearing this mutation to fulfill functional roles in vivo, and hence for the deleterious pathological consequences of the mutation on lens function.
\end{abstract}

(C) 2015 Elsevier B.V. All rights reserved.

\section{Introduction}

Laminins (LM) are highly glycosylated basement membrane proteins built from one $\alpha$, one $\beta$ and one $y$ chain that are linked covalently by disulfide bonds between coiled-coil domains $[2,8,28]$ and assemble into 18 isoforms $[10,17]$. Each subunit comes in a variety of states; there are five isoforms for a (denoted $\alpha 1$ to $\alpha 5$ ), three different types for $\beta$ ( $\beta 1$ to $\beta 3)$ and three variants for $Y(Y 1$ to $Y 3)$ [36]. A common feature of the three types of laminin chains is the presence of an $\mathrm{N}$-terminal short arm containing two globular domains (domains LN (formerly VI) and L4a/LF/L4 (formerly IV)), followed by a series of laminin-type epidermal growth factor-like domains
[3] - as represented schematically in Fig. 1. Exceptions are the laminin $\alpha 4$ and a spliced version of laminin $\alpha 3(\alpha 3 \mathrm{~A})$ that lack the N-terminal short arm [36]. The N-terminal short arms merge into the laminin long arm, a three-stranded left-handed coiled coil. The a laminin chain continues in a tandem array of five laminin globular (LG) domains after the coil [7]. Basement membrane assembly begins with the polymerization of laminin into a cell-associated network [58]. A key step in this process that is mediated by the $\mathrm{N}$-terminal domains of the three short chains of the laminin $\alpha \beta \gamma$ heterotrimer has been described as the "three arms interaction model" [17]. The current work focuses on two truncated forms of the $\mathrm{N}$-terminal 


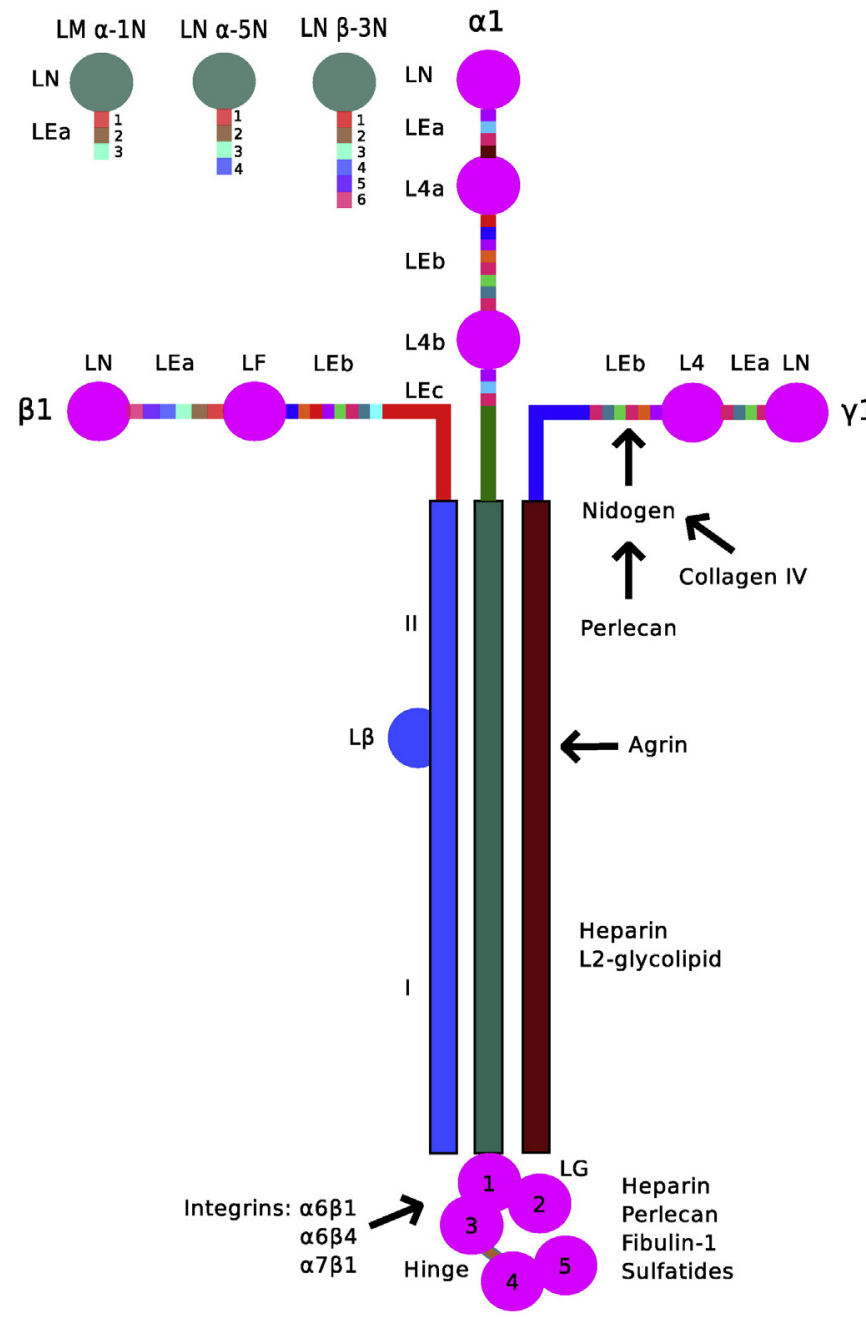

Fig. 1. Schematic representation of the human $\alpha \beta y$ heterotrimer showing the location of the three short-arm segments (LM $\alpha-1 \mathrm{~N}, \mathrm{LM} \alpha-5 \mathrm{~N}$ and LM $\beta-3 \mathrm{~N}$ ) being investigated. region of the LM $\alpha$-short arm, designated LM $\alpha-1 \mathrm{~N}$ and LM $\alpha-5 \mathrm{~N}$, that comprise the globular LN domain and three $(\alpha-1)$ or four ( $\alpha-5)$ LEa domains, and also on a corresponding segment of the LM $\beta$-short arm, (designated LM $\beta-3 \mathrm{~N}$ ), that includes the globular LN domain and six LEa domains. Their location within the laminin $\alpha \beta y$-heterotrimer is indicated in Fig. 1.

Interest in LM $\alpha-1$ stems from its involvement in a number of physiological and pathological processes [10]. Ning et al. [38] demonstrated that the absence of LM $\alpha-1$ results in increased proliferation of mesangial cells in the kidney and increased TGF- $\beta 1$ mediated Smad2 phosphorylation. LM $\alpha-1$ is required for the development and organization of the cerebellum and for the migration of granular cells $[16,21]$. An ablation of $\operatorname{LM} \alpha-1$, which is an essential component of laminin-111 heterotrimer that forms a highly specialized and thick extra-embryonic Reichert's membrane is embryonic lethal [35]. The subunit also regulates neuronal polarity and directional guidance [56], and is required for lens development in zebrafish [60]. A mutation in C56 to serine of LM $\alpha-1 \mathrm{~N}$ in zebrafish leads to defects with the development of lens, cornea, and retina resulting in lens degeneration and focal cornea dysplasia - a mutation also causes death of larvae by 12 days [50]. This cysteine and other cysteine residues are conserved across species as signified by sequence alignment (Supplementary Fig. 1).

LM $\alpha-5$ influences several biological processes including tissue patterning, organogenesis and embryogenesis, and its absence has been linked to limb defects in mouse [52]. Recently, its role in mouse placental labyrinth development and formation has been demonstrated [25]. It is also crucial for the establishment and maintenance of the glomerular filtration barrier in murine kidneys [15,34], as well as for murine lung development [37]. Overexpression of LM $\beta-3$ in colorectal cancer has been linked with chemoresistance of cancer patients [12]. 
A

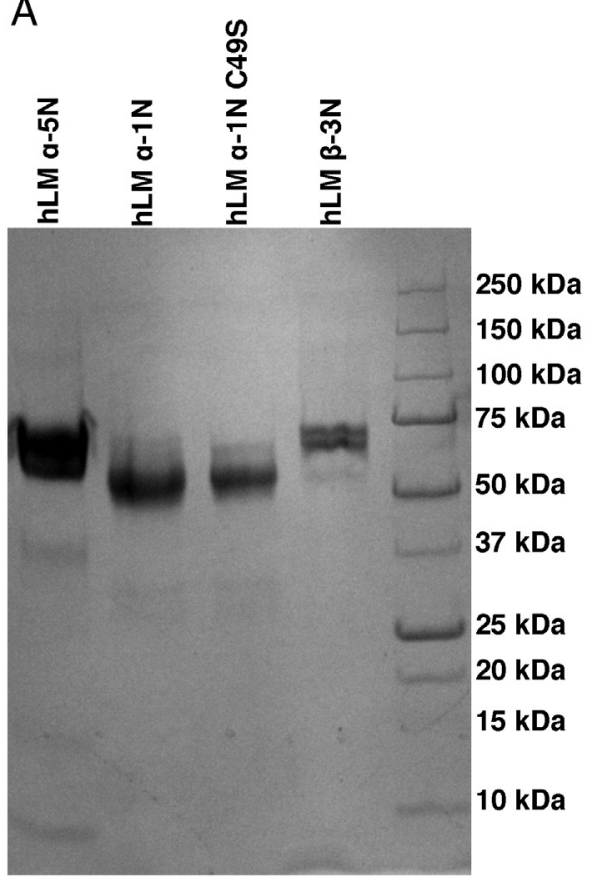

B

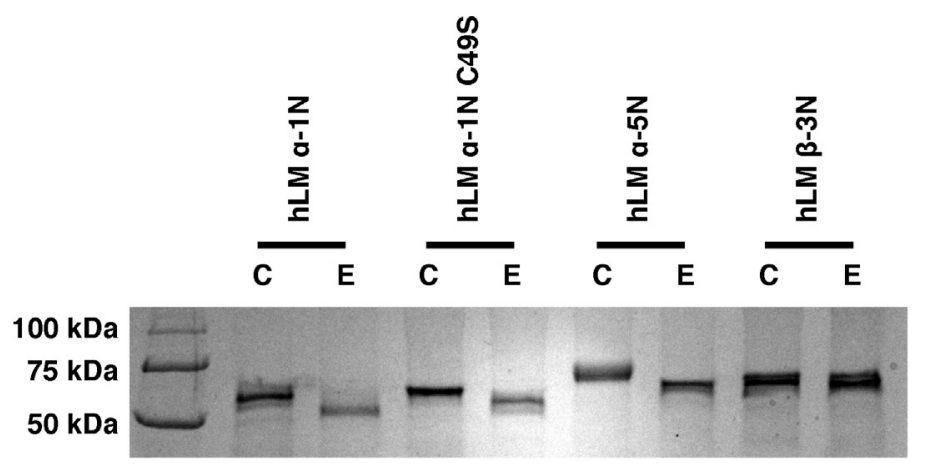

Fig. 2. SDS-PAGE in tricine buffer (A) SDS-PAGE analysis of purified proteins indicating their purity and (B) deglycosylation study of LM $\alpha-1 \mathrm{~N}, \mathrm{LM} \alpha-1 \mathrm{~N}$ C49S, LM $\alpha-5 \mathrm{~N}$ and LM $\beta-3 \mathrm{~N}$ by PNGase F digestion. For each laminin fragment, the gel on the left refers to untreated protein $(C)$, and that on the right to a sample subjected to deglycosylation by PNGase F (E).

The E210K mutation in LM $\beta-3$ leads to a junctional epidermolysis bullosa [33] which was later shown to be rescued by supplementing wild-type LM $\beta-3$ [45].

In view of their structure similarities and involvement with other laminin chains in vivo to form the laminin $\alpha \beta y$-heterotrimer (Fig. 1), some selfassociation of isolated $\mathrm{N}$-terminal fragments of the LM $\alpha-1$ chain might reasonably be expected to occur in the absence of their partners; but on this point there is conflicting evidence. The earlier evidence of such self-association obtained from surface plasmon resonance studies of murine LM $\alpha-1 \mathrm{~N}$ [40] has been refuted in a subsequent size-exclusion chromatography study [44]. Here, we present the results of physicochemical studies on the human $L M \alpha-1 \mathrm{~N}$, LM $\alpha-5 \mathrm{~N}$ and LM $\beta-3 \mathrm{~N}$ that support the earlier finding [40] by revealing a common tendency of these genetically engineered $\mathrm{N}$-terminal regions of short arms to self-associate. The dynamic light scattering (DLS), analytical ultracentrifugation (AUC) and surface plasmon resonance (SPR) data have also suggested that the extent of selfassociation is enhanced by incorporating the equivalent of the deleterious C56S mutation of zebrafish [50], thereby suggesting that elimination of the C56-C72 disulfide bridge (corresponding to C49-C65 in human LM $\alpha-1 \mathrm{~N}$ ) induces a structural change that in turn may prevent proper assembly of laminin heterotrimers containing the $L M \alpha-1$ chain and therefore its export.

\section{Results and discussion}

As a prelude to our hydrodynamic studies, we checked the size and purity of expressed and purified preparations of recombinant $\mathrm{N}$-terminal domains of human laminin short arms, $h L M \alpha-1 N$, hLM $\alpha-5 \mathrm{~N}$, and hLM $\beta-3 \mathrm{~N}$ (Fig. 1) by SDS-PAGE. We also included a mutant form of the hLM $\alpha-1 \mathrm{~N}$ short arm with serine substituted for cysteine 49 (C49S) in our study because of the deleterious effect of the corresponding mutation (C56S) on laminin function in zebrafish [50].

\subsection{Extent of laminin short arm glycosylation}

We examined the extent of short-arm $\mathrm{N}$-glycosylation by monitoring the effect of PNGase $\mathrm{F}$ treatment on the molecular mass deduced from SDS-PAGE (Fig. 2). For the native and C49S mutant forms of hLM a-1 N the deglycosylation treatment led to faster migration, a finding consistent with the presence of 2 potential sites for glycosidic attachment; and a similar situation applies to hLM $\alpha-5$ ( 5 potential sites). However, from the corresponding studies of the hLM $\beta-3 N$ variant it 
Table 1. Effect of deglycosylation on the molecular mass of laminin fragments.

\begin{tabular}{|c|c|c|c|c|c|c|}
\hline \multirow[t]{2}{*}{ Species } & \multicolumn{4}{|c|}{ Molecular mass (kDa) } & \multicolumn{2}{|c|}{ Partial specific volume $(\mathrm{mL} / \mathrm{g})$} \\
\hline & Polypeptide $^{*}$ & Untreated $^{\dagger}$ & Deglycosylated & N-Glycan sites ${ }^{\#}$ & Polypeptide $^{*}$ & Glycoprotein \\
\hline hLM $\alpha-1 \mathrm{~N}$ & 55.2 & 57 & 52 & 2 & 0.718 & 0.715 \\
\hline hLM a-1 N C49S & 55.2 & 57 & 52 & 2 & 0.718 & 0.715 \\
\hline hLM $\alpha-5 \mathrm{~N}$ & 61.6 & 73 & 61 & 5 & 0.712 & 0.709 \\
\hline hLM $\beta-3 \mathrm{~N}$ & 67.5 & 69 & 69 & 1 & 0.708 & - \\
\hline \multicolumn{7}{|c|}{$\begin{array}{l}\text { * Value calculated from the amino acid sequence. } \\
\dagger \text { SDS-PAGE estimate. } \\
\# \text { From NetNGlyc } 1.0 \text { Server (http://www.cbs.dtu.dk/services/NetNGlyc/). } \\
\ddagger \text { Calculated from Eq. (1). }\end{array}$} \\
\hline
\end{tabular}

would appear that this part of the laminin heterotrimer might not be glycosylated (1 potential site). Inspection of Table 1 reveals that deglycosylation has decreased the apparent molecular masses $(M)$ of the $h L M \alpha-1 \mathrm{~N}$ and $\mathrm{hLM} \alpha-5 \mathrm{~N}$ to values more in keeping with those calculated from the amino acid sequences. On the other hand, the general agreement between all three estimates of the molecular mass $M$ for the hLM $\beta-3 \mathrm{~N}$ confirms the essential absence of glycosidic modification on this polypeptide.

Also shown in Table 1 are values of the partial specific volume $(\bar{v})$ calculated for the polypeptide and the glycoprotein - parameters required for molecular mass estimation from sedimentation velocity and dynamic light scattering studies. In that regard the value for the glycoprotein $\left(\bar{v}_{G P}\right)$ has been calculated from the expression

$$
\bar{v}_{G P}=\left(M_{P} \bar{v}_{P}+\left(M_{G P}-M_{P}\right) \bar{v}_{C}\right) / M_{G P}
$$

where $M_{G P}$ refers to the SDS-PAGE estimate of molecular mass for the glycoprotein, and $M_{P}$ to the calculated value for the undecorated polypeptide: $\bar{v}_{P}$, the polypeptide partial specific volume, has been calculated from the amino acid composition by the program SEDNTERP [30] and a value of $0.63 \mathrm{~mL} / \mathrm{g}$ has been assigned to $\bar{v}_{C}$, the partial specific volume of the carbohydrate decoration. Despite being significant, the extent of glycosylation of the $h L M$ a-1 $\mathrm{N}$ and hLM $\alpha-5 \mathrm{~N}$ only has a minor influence on partial specific volume (final two columns of Table 1).

\subsection{Evidence for secondary structure differences between laminin fragment variants}

Differences in secondary structure between the laminin fragment variants have been revealed by circular dichroism (CD) studies. All of the spectra exhibit a distinctive minimum at $206 \mathrm{~nm}$ and a maximum or shoulder at about $196 \mathrm{~nm}$ (Fig. 3) features that signify the existence of $\alpha$-helical and/or $\beta$-pleated sheet regions within the three-dimensional protein structures. However, there are quantitative differences between the secondary structures of $h L M \alpha-1 \mathrm{~N}, \mathrm{hLM} \alpha-5 \mathrm{~N}$ and $\mathrm{hLM} \beta-3 \mathrm{~N}$. In that regard the nearly identical forms of the CD spectra of

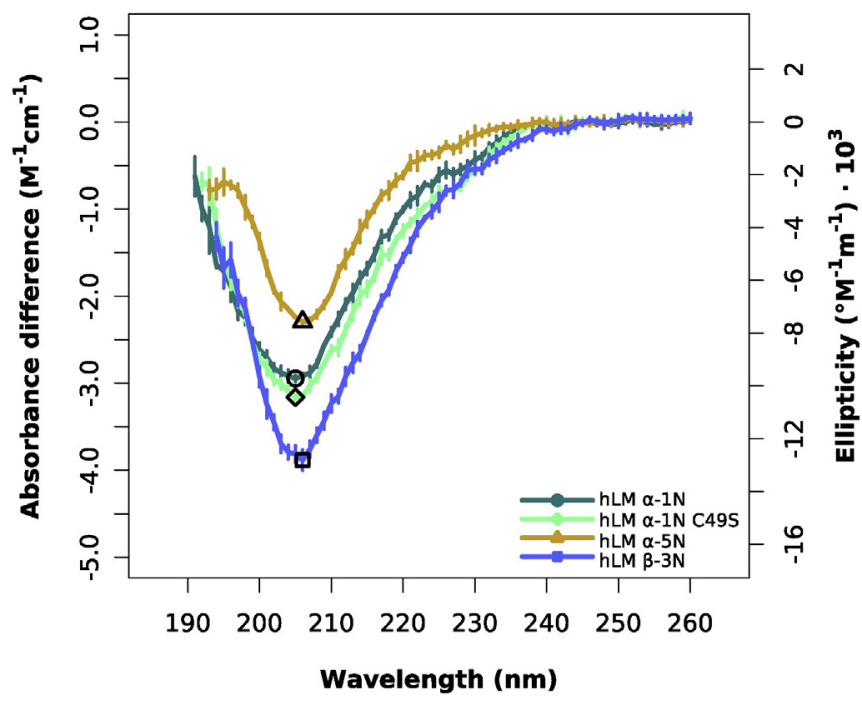

Fig. 3. CD spectra for $1.2 \mathrm{~g} / \mathrm{L} h L M \alpha-1 \mathrm{~N}(\Delta)$, $1.1 \mathrm{~g} / \mathrm{L}$ hLM $\alpha-1 \mathrm{~N}$ C49S mutant $(\nabla), 1.3 \mathrm{~g} / \mathrm{L}$ hLM $\alpha-5 \mathrm{~N}(\square)$, and $1.1 \mathrm{~g} / \mathrm{L} \mathrm{hLM} \beta-3 \mathrm{~N}(\diamond)$ in Tris-chloride buffer $(\mathrm{pH} 8.5, \mid \mathrm{I} 0.17 \mathrm{M})$. Vertical lines denote the standard deviations of three independent measurements. The figure was generated using the program $R$ [61]. 


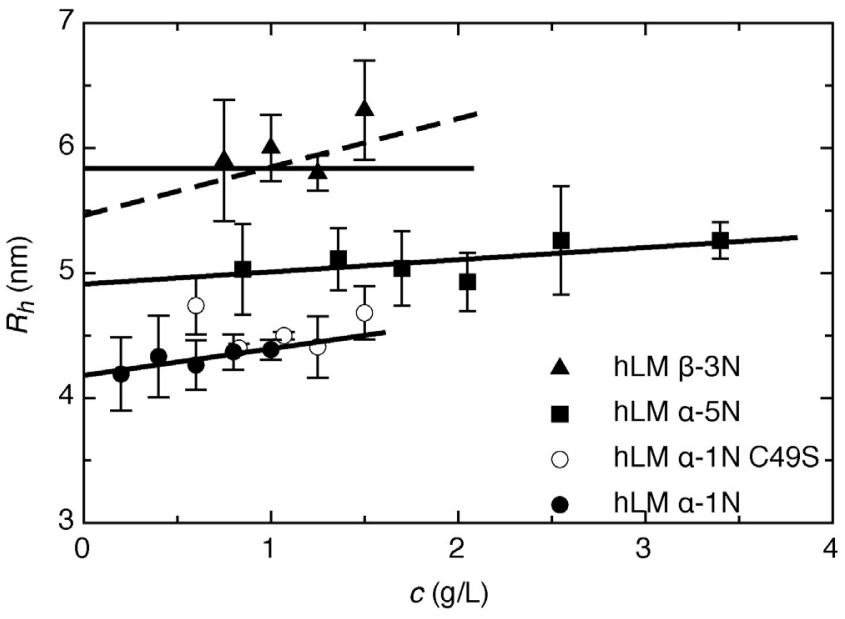

Fig. 4. Concentration dependence of hydrodynamic radii determined by dynamic light scattering measurements on solutions of $\mathrm{hLM} \alpha-1 \mathrm{~N}(\bullet)$ and its C49S mutant $(\circ)$, hLM $\beta-3 \mathrm{~N}(\mathbf{\Delta})$ and $\mathrm{hLM} \alpha-5 \mathrm{~N}(\boldsymbol{\bullet})$ in Trischloride buffer ( $\mathrm{pH} 8.5, \mathrm{I} 0.17 \mathrm{M})$. wild-type hLM $\alpha-1 \mathrm{~N}$ and its C49S mutant signify that the mutation has not induced a significant change in the secondary structure content.

\subsection{Reversible self-association of short arm fragments}

Indirect evidence for the self-association of laminin short arm fragments was obtained by Odenthal et al. [40], who employed surface plasmon resonance to demonstrate interaction of fragments with immobilized partners on a biosensor chip. However, this finding conflicts with those from a subsequent study [44] in which size exclusion chromatography (SEC) was used to investigate the self-association of a species in solution rather than its interaction with a chemically modified (immobilized) counterpart. Here we employ two additional procedures, dynamic light scattering (DLS) and sedimentation velocity ultracentrifugation, to comment further on the macromolecular state of laminin short arm fragments in solution.

Although the quantity monitored in DLS is the translational diffusion coefficient $(D)$, the Zetasizer software supplied with the Malvern instrument employs the Stokes-Einstein relationship to convert $D$ into the Stokes radius $R_{h}$, i.e., the radius of an equivalent hydrodynamic sphere. Previous studies have shown that a stable, nonassociating species exhibits a negative concentration dependence of $R_{h}$ [48], which accords with positive concentration dependence of the diffusion coefficient measured by DLS [22]. In compliance with the minimum requirement for solute homogeneity with respect to molecular size, the volume weighted Stokes radius distributions for laminin fragments in Tris-chloride buffer, pH 8.5, I 0.17 M (see Materials and methods for composition), were invariably unimodal and symmetrical. However, as is evident from Fig. 4, the values of $R_{h}$ (in $\mathrm{nm}$ ) deduced from the peaks for many of these distributions exhibit a positive dependence upon concentration $(c)$ - the characteristic of a solute species undergoing rapid reversible self-association. For example, the DLS results for wild-type hLM $\alpha-1 \mathrm{~N}(\bullet)$ conform with the linear relationship $R_{h}=4.20( \pm 0.05)+0.22( \pm 0.08) c$ $(-)$, which also provides a reasonable description of results for the C49S mutant (O). Similarly, a linear dependence, $R_{h}=4.9( \pm 0.1)+0.10( \pm 0.05) c$, describes the results for hLM $\alpha-5 N(\square)$. Although the corresponding best-fit description $\left(-{ }^{-}\right)$of the hLM $\beta$-3 N data ( $\mathbf{\Lambda})$ would suggest positive concentration dependence, no significance can be attached to the slope because the uncertainty $( \pm 1 S D)$ therein matches its magnitude. Therefore, these results have been averaged to obtain an estimated hydrodynamic radius of $6.2( \pm 0.2) \mathrm{nm}$ for the $\mathrm{hLM} \beta-3 \mathrm{~N}$ (solid line through the data in Fig. 4).

Analyses of sedimentation velocity distributions essentially echo the DLS findings in that the observation of a progressive shift of the $c(s)$ distributions $[9,47]$ with increasing hLM $\alpha-1 \mathrm{~N}$ concentration (Fig. 5A) also signifies the selfassociation of this fragment under the current conditions ( $\mathrm{pH} 8.5, \mathrm{I} 0.17 \mathrm{M})$ : an estimate of 3.27 $( \pm 0.03)$ for $s_{20, w}^{0}$ is obtained from the ordinate intercept of an essentially linear concentration dependence of $s_{20, w}^{O}$ (inset to Fig. 5A). Combination of this value of $S_{20, w}^{O}$ with the corresponding limiting hydrodynamic radius $\left(R_{h}^{o}\right)$ of $4.2( \pm 0.05) \mathrm{nm}$ (Fig. 4) in the expression

$$
M=6 \pi N_{A} \eta_{20, w} R_{h}^{o} s_{20, w}^{o} /\left(1-\bar{v} \rho_{20, w}\right)
$$


A

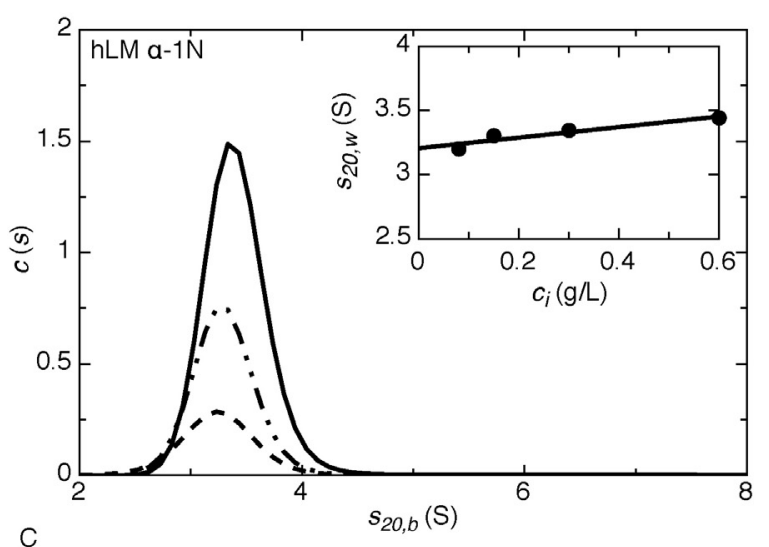

C

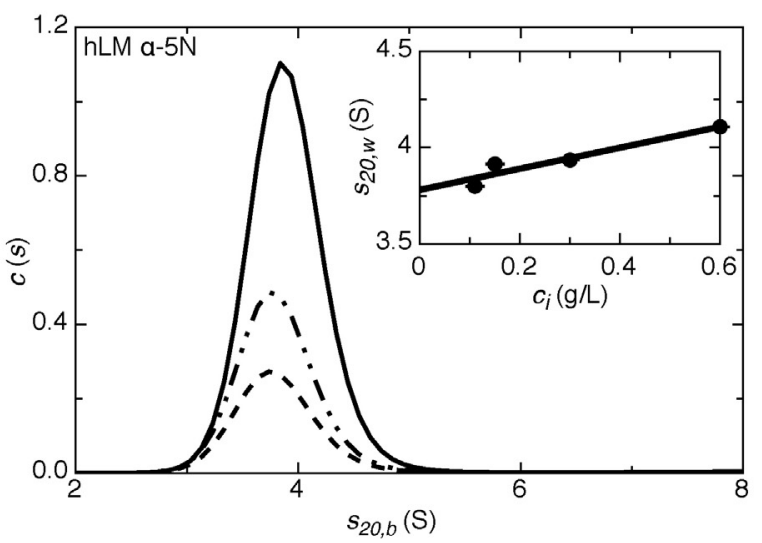

B
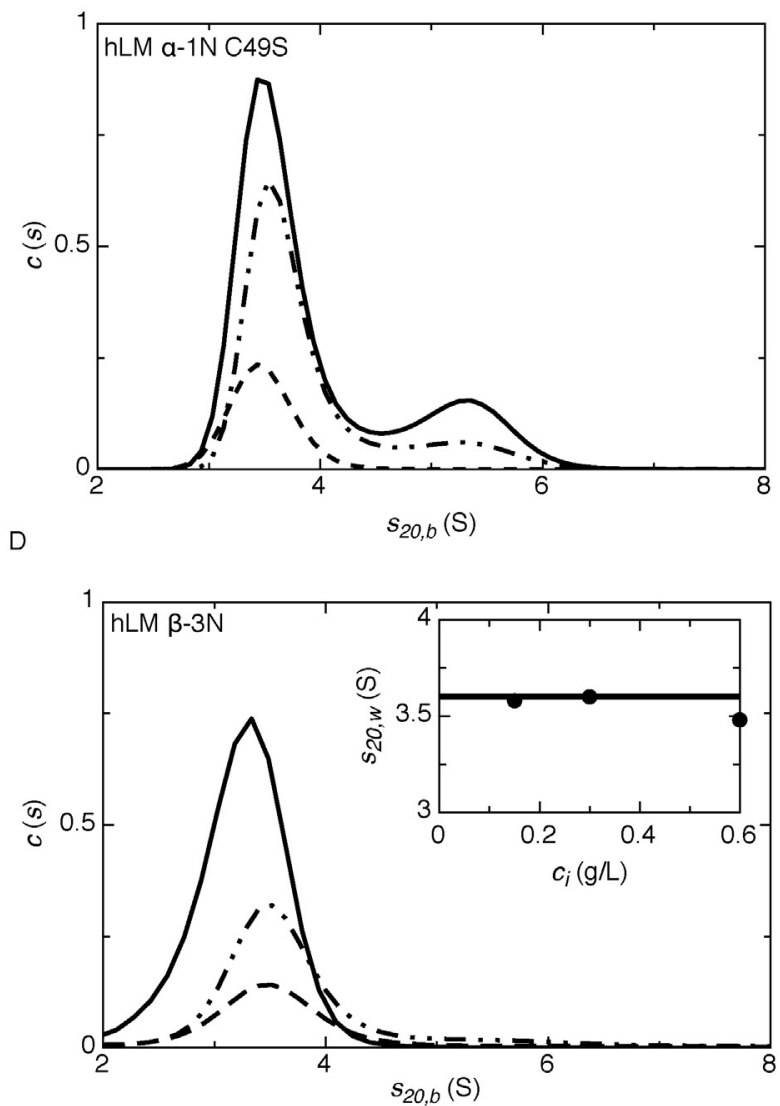

Fig. 5. Analytical ultracentrifugation studies of laminin short arm fragments. (A) SEDFIT analysis $[9,47]$ of sedimentation velocity distributions for different concentrations of $(A) \mathrm{hLM} \alpha-1 \mathrm{~N}$ and $(B)$ its C49S mutant in Tris-chloride buffer (pH 8.5, I $0.17 \mathrm{M}):-, 0.6 \mathrm{~g} / \mathrm{L} ;----, 0.3 \mathrm{~g} / \mathrm{L} ;---, 0.15 \mathrm{~g} / \mathrm{L}$. Inset: concentration dependence of the derived sedimentation coefficient for hLM a-1 N. (C) SEDFIT analysis of sedimentation velocity distributions for $0.6 \mathrm{~g} / \mathrm{L}(-), 0.3 \mathrm{~g} / \mathrm{L}(----)$, and $0.15 \mathrm{~g} / \mathrm{L}(---)$ solutions of $\mathrm{hLM} \alpha-5 \mathrm{~N}$ in Tris - chloride buffer $(\mathrm{pH} 8.5, \mathrm{I} 0.17 \mathrm{M})$, together with the concentration dependence of the derived sedimentation coefficients (inset). (D) SEDFIT analysis of sedimentation velocity distributions for $0.6 \mathrm{~g} / \mathrm{L} \mathrm{(-)}$, $0.3 \mathrm{~g} / \mathrm{L}\left(--_{--}\right)$, and $0.15 \mathrm{~g} / \mathrm{L}(---)$ solutions of hLM $\beta-3 \mathrm{~N}$ in Tris-chloride buffer (pH 8.5, I 0.17 M), together with the concentration dependence of the derived sedimentation coefficients (inset).

[where $\eta_{20, w}$ and $\rho_{20, w}$ are the viscosity and density, respectively, of water at $20^{\circ} \mathrm{C}$; and where $N_{A}$ is Avogadro's number] yields a molecular mass of 56 $( \pm 1) \mathrm{kDa}$ that matches the estimate of $57 \mathrm{kDa}$ obtained by SDS-PAGE (Table 1).

The quantitative similarity observed between the concentration dependencies of $R_{h}$ distributions for wild-type hLM $\alpha-1 \mathrm{~N}$ and the C49S mutant (Fig. 4) does not extend to their sedimentation coefficient behavior (Fig. 5B). Although the mutant hLM a-1 N also exhibits a positive dependence of $s$ on $c_{i}$ that is commensurate with rapid self-association, the presence of a second peak implicates participation of a larger oligomeric state in the association phenomenon. Whereas a unimodal reaction boundary (as in Fig. 5A) is the predicted outcome of rapid, reversible dimerization, bimodality of the reaction boundary can be encountered with systems exhibit- ing self-association beyond dimer [14]. Indeed, the $C(s)$ profiles presented in Fig. 5B resemble those predicted boundary forms. At low concentrations the predicted pattern is a unimodal reaction boundary with a sedimentation coefficient approximating that of monomer, the upper-limiting size of which is governed by the stoichiometry $(n)$ and strength of self-association. For a two-state self-association $\left(n \mathrm{~A} \leftrightarrows \mathrm{A}_{n}\right)$ with $n$ greater than 2 , the predicted consequence of increasing the solute concentration is thus the appearance of a second, partially resolved reaction boundary whose area and migration rates both increase with concentration. Although the patterns in Fig. 5B are qualitatively consistent with such predicted behavior, the faster peak could conceivably reflect stable dimer formation via a disulfide bridge between the unpaired cysteine residue in monomers of the C49S mutant - a 
A

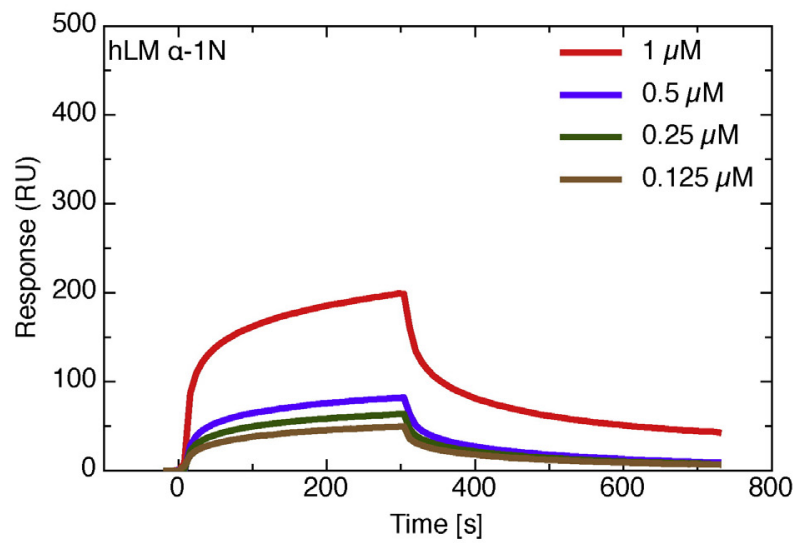

$\mathrm{B}$

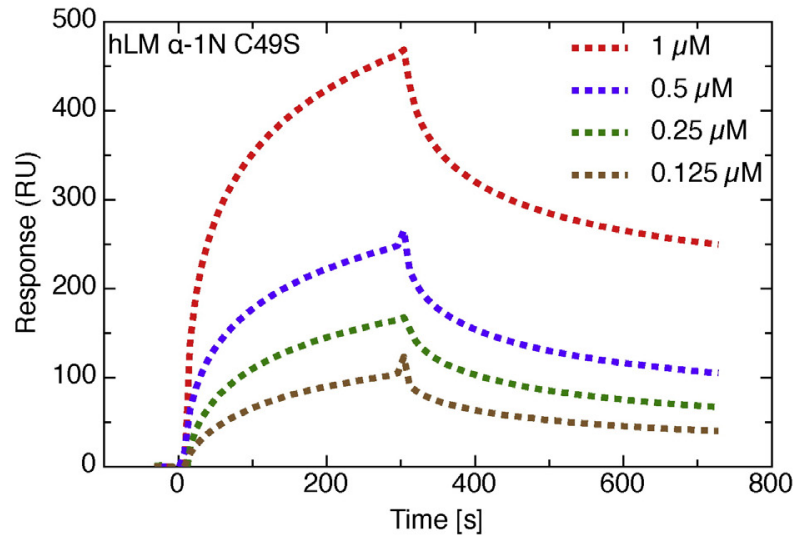

C

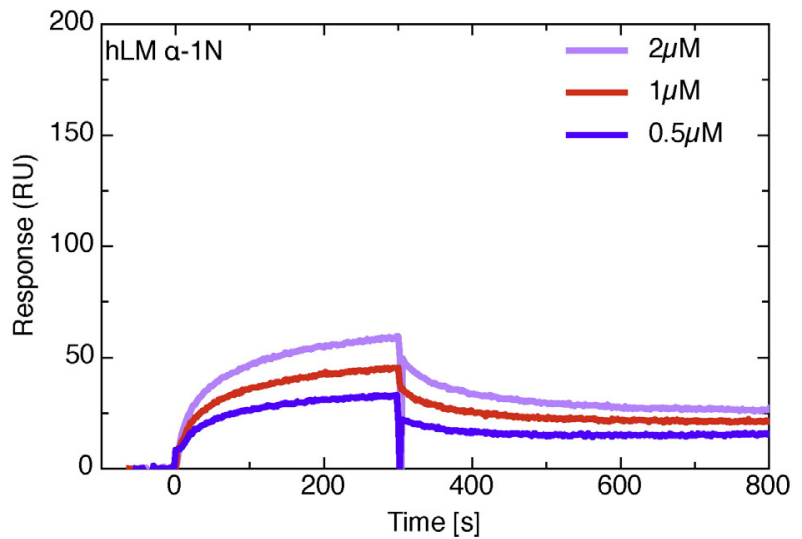

D

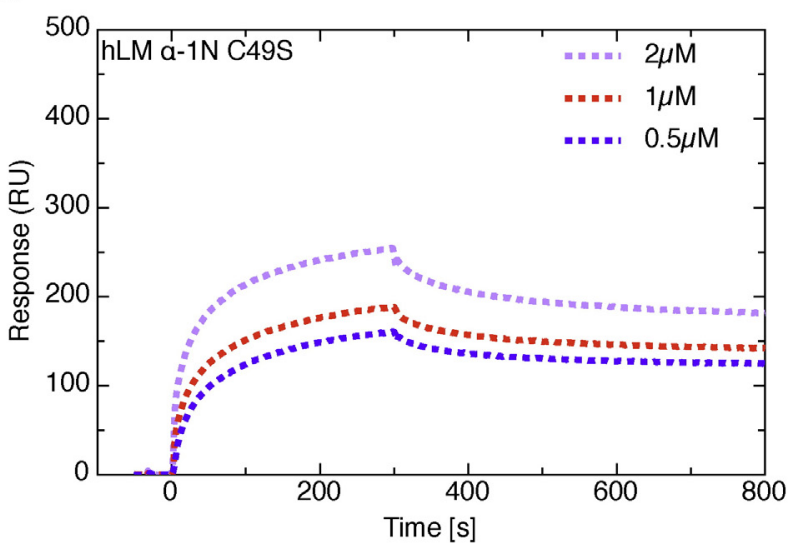

Fig. 6. Evidence obtained by SPR studies supporting the concept of hLM $\alpha-1 \mathrm{~N}$ and $\alpha-1 \mathrm{~N}$ C49S self-association. (A) Sensorgrams showing the adsorption and desorption stages of hLM $\alpha-1 \mathrm{~N}$ wild-type interaction with its immobilized counterpart in the absence of $\mathrm{Ca}^{2+}: k_{a}=1.8 \times 10^{4} \mathrm{M}^{-1} \mathrm{~s}^{-1} ; k_{d}=3.6 \times 10^{-3} \mathrm{~s}^{-1}\left[X^{2}=0.49\right]$. (B) Corresponding profiles for hLM a-1 N C49S with its immobilized counterpart under the same conditions: $k_{a}=2.3 \times 10^{4} \mathrm{M}^{-1} \mathrm{~s}^{-1} ; k_{d}=$ $1.7 \times 10^{-3} \mathrm{~s}^{-1}\left[x^{2}=0.65\right]$. (C) Sensorgrams for hLM $\alpha-1 \mathrm{~N}$ wild-type in the presence of $2 \mathrm{mM}^{\mathrm{Ca}} \mathrm{Ca}^{2+}: k_{a}=$ $1.1 \times 10^{4} \mathrm{M}^{-1} \mathrm{~s}^{-1} ; k_{d}=1.3 \times 10^{-3} \mathrm{~s}^{-1}\left[X^{2}=0.14\right]$. (D) Corresponding patterns for the hLM $\alpha-1 \mathrm{~N}$ C49S mutant under the same conditions: $k_{a}=1.6 \times 10^{4} \mathrm{M}^{-1} \mathrm{~s}^{-1} ; k_{d}=4.8 \times 10^{-4} \mathrm{~s}^{-1}\left[X^{2}=0.81\right]$.

possibility seemingly precluded by the essential identity of SDS-PAGE profiles under reducing and non-reducing conditions (Supplementary Fig. 2). In either event the C56S mutation has introduced a second mode of self-association involving a higher oligomeric state.

The sedimentation velocity distributions for wildtype hLM $\alpha-5 \mathrm{~N}$ resemble qualitatively their counterparts for wild-type hLM $\alpha-1 \mathrm{~N}$ by being unimodal and exhibiting positive $s-c$ dependence (Fig. 5C). Combination of the extrapolated value of 3.82 $( \pm 0.05) S$ for $S_{20, w}^{o}$ with the corresponding hydrodynamic radius $\left(R_{h}^{o}\right)$ of $4.9( \pm 0.1 \mathrm{~nm})$ in Eq. (2) yields a monomer molecular mass of $74( \pm 2) \mathrm{kDa}$, which is again in reasonable agreement with the SDS-PAGE estimate of $73 \mathrm{kDa}$ for the glycoprotein.

In keeping with the DLS results for hLM $\beta-3 N$ (Fig. 2B) the unimodal sedimentation velocity distributions for this species (Fig. 5D) exhibit little concentration dependence. However, the asymmetry of the profile at the highest protein concentration $(0.6 \mathrm{~g} / \mathrm{L})$ is a predicted characteristic of the reaction boundary for a reversibly associating protein [14]. Qualitative support for the concept that $h L M \quad \beta-3 N$ may also undergo self-association (albeit weaker than that for the other laminin short arms) comes from the combination of the average value of 3.56 $( \pm 0.12) \mathrm{S}$ for $S_{20, w}$ with the average Stokes radius of $6.0( \pm 0.2) \mathrm{nm}$ to obtain a molecular mass estimate of $85( \pm 6) \mathrm{kDa}$ - a value much higher than the sequence value of $68 \mathrm{kDa}$ for this unglycosylated polypeptide chain (Table 1). Consequently, despite failure to observe the positive $s-c$ dependence that allowed unequivocal identification of the hLM a-1 N and $\mathrm{hLM} \alpha-5 \mathrm{~N}$ as self-associating systems, we conclude that the hLM $\beta-3 \mathrm{~N}$ may also undergo weak self-association under the same buffer conditions ( $\mathrm{pH} 8.5$, I $0.17 \mathrm{M})$. 
The present hydrodynamic evidence for the selfassociation of laminin short arm fragments needs to be reconciled with an earlier report [44] that disputed its existence - a claim based on the observation that individual short arm fragments (approx. $1 \mathrm{mg} / \mathrm{mL}$ ) eluted as single monomeric peaks in zonal size-exclusion chromatography on Superdex 200. Such failure to detect any selfassociation reflects the insensitivity of zonal sizeexclusion chromatography because of the progressive dilution and hence oligomer dissociation that occurs during passage of the reaction zone down the column [5,53]. Indeed, no concentration dependence of $R_{h}$ or $s_{20, w}$ would have been detected in the present study at concentrations of $0.1 \mathrm{mg} / \mathrm{mL}$ or lower (Figs. 4 and 5) - values likely to be pertinent to the elution profiles shown in Fig. 2A of Purvis and Hohenester [44]. A more definitive assessment of the self-association characteristics would have been obtained by frontal size-exclusion chromatography [54].

\subsection{Comparison of wild-type and mutant hLM $\alpha-1 \mathrm{~N}$ self-association by SPR}

In these surface plasmon resonance experiments hLM $\alpha-1 \mathrm{~N}$ and its C49S mutant were coupled to separate NTA-chips via their His tags in order to ensure a uniform orientation of immobilized ligand on the biosensor surface. The consequent advantage of uniform ligand orientation is, of course, offset by the need for elimination of metal ions from the applied analyte solutions (by the inclusion of EDTA) to avoid displacement of immobilized ligand from the sensor chip. In view of the demonstrated inhibitory effect of $\mathrm{Ca}^{2+}$ removal on the interaction between laminin short arm fragments [40], SPR experiments with $\mathrm{hLM} \alpha-1 \mathrm{~N}$ attached in random orientation to a CM5 chip were also performed to ascertain that qualitatively similar results were obtained in the $\mathrm{Ca}^{2+}$ containing buffer used for all of the solution studies.

The SPR time-courses presented in Fig. $6 \mathrm{~A}$ and $\mathrm{B}$ reflect experiments entailing the passage of a range of concentrations of laminin short arm fragment over NTA biosensor chips to which the same fragment had been attached. For either laminin short arm fragment (wild-type or mutant) the flow of analyte across the biosensor chip leads to a fairly rapid increase in response, and a corresponding decrease upon substitution of buffer as the flowing solution. Despite the absence of $\mathrm{Ca}^{2+}$ ion in these SPR experiments, the traces presented in Fig. 6 attest to rapid and reversible interaction of soluble fragment with its immobilized counterpart on the sensor chip. Routine analysis of those time-courses in terms of 1:1 stoichiometry [39]yields apparent dissociation constants of $200 \mathrm{nM}$ and $74 \mathrm{nM}$ for the uptake of wild-type and mutant fragments respectively. Although these values do not refer to the corre- sponding interaction in solution, they do afford a qualitative assessment of relative extents of fragment self-association. This observation of a 3 -fold stronger interaction for the C49S mutant is clearly in keeping with the above sedimentation velocity findings (Fig. 5B).

Similar conclusions stem from the corresponding SPR studies with the NTA biosensor chips replaced by their CM5 counterparts to allow the inclusion of calcium ion $(2 \mathrm{mM})$ in the buffer system. The lower $K_{D}$ of $120 \mathrm{nM}$ for wild-type hLM a-1 N under these conditions (Fig. 6C) signifies enhanced selfassociation in the presence of metal ion; and it must be noted that the ratio of dissociation constants in the presence and absence of $\mathrm{Ca}^{2+}$ underestimates the extent of that enhancement because of a comparison between a $K_{D}$ for interaction with uniquely orientated immobilized ligand in the absence of metal ion and an averaged value obtained in the presence of $\mathrm{Ca}^{2+}$ for the binding of analyte to immobilized ligand in a random array of orientations. These findings are thus consistent with earlier observations that the immobilization of his-tagged short arm fragments to NTA-coupled microspheres for XMAP-Luminex binding assays in the presence of $\mathrm{Ca}^{2+}$ consistently leads to the return of smaller dissociation constants than those obtained by SPR on CM5 sensor chips [see Table 2 of Odenthal et al. [40]].

In keeping with the SPR results obtained for the hLM $\alpha-1 \mathrm{~N}$ mutant in the absence of metal ion, the studies on the CM5 chip have signified tighter interaction between this analyte and its immobilized counterpart in the presence of $\mathrm{Ca}^{2+}$ (Fig. 6D). Indeed, the four-fold enhancement of interaction $\left(K_{D}=30 \mathrm{nM}\right.$ cf $\left.120 \mathrm{nM}\right)$ for the mutant is very similar to the three-fold effect ( $74 \mathrm{nM}$ cf $180 \mathrm{nM}$ ) seen in the metal-free environment. Although such quantitative interpretation of the relative $K_{D}$ values must be qualified by statistical considerations (see the legend to Fig. 6 for $x^{2}$ values), the results presented in Fig. 6 suffice to provide additional qualitative support for the concept of laminin short arm self-association in the absence of components from the other short arms that comprise the $\alpha \beta \gamma$ heterotrimer.

\section{Summary}

Laminin $\alpha$-, $\beta$ - and $\gamma$-chains form a heterotrimeric molecule inside the cell, which is then secreted outside. The laminin N-terminal domains (LN) of $\alpha$-, $\beta$ - and $\gamma$-chains are key components required for further interactions of laminin heterotrimers [32]. The laminin heterotrimer assembly begins with noncovalent but specific interactions between the $C$-terminal long arm of $\beta$ - and $\gamma$-chains $[4,31]$ followed by the formation of disulfide bridges that stabilize the assembly between these two chains $[1,19,27]$. The LM $\alpha$ chain then interacts with the 


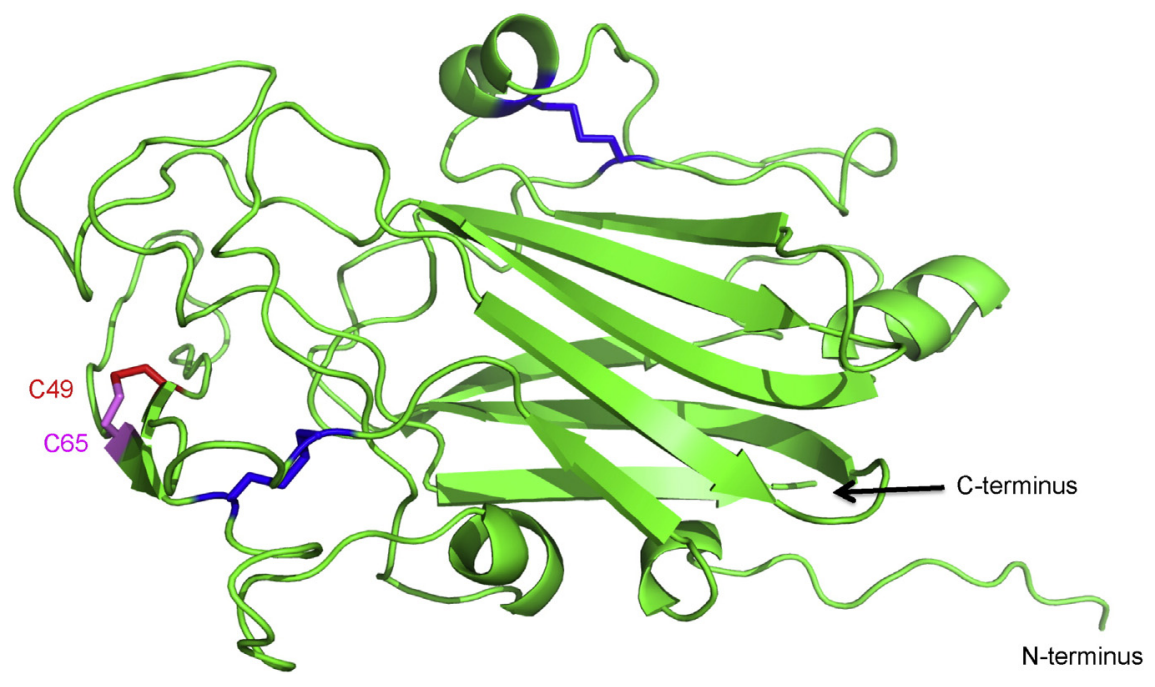

Fig. 7. Homology model of hLM a-1 N-terminal globular domain calculated using Phyre2 [24] server and templates pdb files - 4AQS, 4AQT [6], 4PLO [57], 3ZYJ [49], and 2Y38 [20]. Blue sticks identify two of the disulfide bridges, whereas the third bridge, between C49 (corresponding to C56 in zebrafish) and C65 (corresponding to C72 in zebrafish), is identified by highlighting the respective residues in red and magenta. (For interpretation of the references to color in this figure legend, the reader is referred to the web version of this article.)

complex of LM $\beta$ and $y$ chain that is essential for the secretion of $\alpha \beta \gamma$ heterotrimers $[27,59]$.

Our study reveals hydrodynamic evidence for the self-association of three laminin fragments (hLM $\alpha-1 \mathrm{~N}, \mathrm{hLM} \alpha-5 \mathrm{~N}$ and $\mathrm{hLM} \beta-3 \mathrm{~N}$ ) of the laminin $\alpha \beta \gamma$ heterotrimer short arms. Bioinformatics prediction suggests that the $N$-terminal region of $h L M \quad \beta-3 N$ has a single glycosylation site, compared to hLM a-1 $\mathrm{N}$ and hLM $\alpha-5 \mathrm{~N}$ chains, however we observe no significant difference between PNGaseF digested $\beta-3 \mathrm{~N}$ and undigested $\beta-3 \mathrm{~N}$. Although the glycosylation for $h L M \quad \alpha-1 N$ and $h L M \alpha-5 N$ is confirmed by the PNGaseF digestion (Fig. 2), this difference in extent of post-translational glycan attachment does not seem to play any significant role in self-association of these $\mathrm{N}$-terminal fragments in that $h L M \quad \beta-3 N$ also undergoes weak selfassociation (Fig. 5D). In similar vein the LEa domains do not seem to play a significant role in the self-association process in that $h L M \quad \beta-3 N$ (with 6 LEa domains), hLM $\alpha-1 \mathrm{~N}$ (3 LEa domains), and $\mathrm{hLM} \alpha-5 \mathrm{~N}$ (4 LEa domains all undergo reversible self-association;Fig. 5A, C, D). Such self-association, first detected by SPR studies on murine LM $\alpha-1 \mathrm{~N}$ [40], should not be construed as evidence for the biological significance of this phenomenon, but rather as a consequence of the presence on the monomer surface of amino acid residues with potential for noncovalent interaction with similarly disposed residues on the surface of other monomers. In the current experiments on isolated short arm fragments this additional noncovalent interaction necessarily involves self- association, but in the biological context it would entail heterologous association involving $\alpha, \beta$ and $\gamma$ chains involved in heterogeneous association with similarly disposed regions on the other chains comprising the $\alpha \beta \gamma$ heterotrimer - the feature responsible for their functional roles in the assembly of laminin, its incorporation into the basic membrane structure, and the provision of matrix sites for the attachment of extracellular ligands. In that regard the extended self-association to higher order oligomers that was observed for the C49S mutant of hLM a-1 N signifies structural changes resulting from disruption of the C49-C65 disulfide bridge that may well be related to the inability of laminin bearing the corresponding mutation (C56S) to fulfill those functional roles in zebrafish, and hence to the deleterious pathological consequences of the mutation on lens function $[43,50]$.

Apart from stabilizing the interactions between LM $\beta$ and $y$ chains, the disulfide bonds are also crucial to maintain the tertiary structure of the globular LN domains located at the $\mathrm{N}$-terminus of individual chains. Therefore, the deleterious pathological consequences in zebrafish may, of course, also reflect the disruption of a disulfide bridge that is important for the tertiary structure of LN domain. Because there is currently no high-resolution information available for the hLM $\alpha-1 \mathrm{~N}$, homology models calculated with Phyre2 [24], HHpred [51] and M4T [11] servers and template pdb files - 4AQS [6], 4PLO [57], 3ZYJ [49], and $2 Y 38$ [20], have been used to reveal that the cysteine residue in question (C49) is involved in one of three disulfide bridges in the LN domain. Fig. 7 
presents that homology model for hLM $\alpha-1 \mathrm{~N}$ (Phyre2 program) displaying two disulfide bridges in blue as well as the third one between C49 (red) and C65 (magenta). Mutation of C49 to S49 not only disrupts the bridge between C49 and C65 but may also introduce structural changes that alter the distribution of hydrophobic groups on the monomer surface, and lead to enhance hydrophobic self-association to potential for interaction between adjacent hLM $\alpha-1 \mathrm{~N}$ monomers. The mutation-induced enhancement of $\mathrm{hLM} \alpha-1$ self-association is therefore regarded as an indicator of the change in monomer tertiary structure that may inhibit its incorporation into the laminin $\alpha \beta y$ heterotrimer. The embryonic lethality due to mutations in $L M \alpha, \beta$ and $y$ chains has been previously reported $[18,23,36]$. Since laminin 111 is the first laminin heterotrimer found during embryogenic development and a key component for the basement membrane assembly that affects a number of cellular signaling pathways, it is not surprising that mutations such as LM $\alpha-1$ C49S (C56S in zebrafish) affecting laminin 111 functions lead to embryogenic lethality. However, it is still open whether laminin 111 bearing the C49S mutation exhibit an altered secretion due to inability to form this laminin heterotrimer or whether it inhibits proper laminin assembly into a polymer-like sheet.

\section{Materials and methods}

\subsection{Expression and purification of $\mathrm{N}$-terminal fragments of recombinant human laminin short arms}

The corresponding coding sequences of the human laminin N-terminal domains of $\alpha-1, \alpha-5$ and $\beta-3$ chains, LM $\alpha-1 \mathrm{~N}$ (wild-type and C49S mutant), LM $\alpha-5 \mathrm{~N}$ and LM $\beta-3 \mathrm{~N}$ were amplified by PCR from different human cDNAs. The following primers were used: human LM $\alpha-1$ forward 5 '-aaagctagccggcagagaggectgtttcctg- $3^{\prime}$ and reverse $5^{\prime}$-tttggatccttaggagacacaggtcgggt- $3^{\prime}$; human LM $\alpha-5$ forward $5^{\prime}$-aaagc tagcacgggaggaggcgggeggcggct- $3^{\prime}$ and reverse $5^{\prime}$-ttt ggatccgggetggcagccggggccgtaga- $3^{\prime}$; human LM $\beta-3$ forward $5^{\prime}$-acagctagcacaacaagcctgctcccgtggg-3' and reverse $5^{\prime}$-tttagatctggctcggcatcctgtggccacg-3'. The C49S mutation was introduced into the LM a-1 coding sequence by overlap PCR. Each construct was cloned into a modified pCEP-Pu expression vector carrying a $5^{\prime}$-BM40 signal and a C-terminal double strep II tag. The sequenced plasmid were transfected into HEK 293 cells and all four laminin $\mathrm{N}$-terminal fragments were then purified from the collected cell culture supernatants by affinity chromatography on a Strep-Tactin Sepharose column (IBA, Germany) using $2.5 \mathrm{mM}$ desthiobiotin in $40 \mathrm{mM}$ Tris $/ \mathrm{HCl}(\mathrm{pH} 8.0)$ supplemented with
$150 \mathrm{mM} \mathrm{NaCl}$ as eluent. After evaluating the purity of each laminin fragment by SDS-PAGE in Tricine buffer [46], the purified preparations of $h L M \alpha-1 \mathrm{~N}$ (wild-type and C49S mutant), hLM $\alpha-5 \mathrm{~N}$ and hLM $\beta-3 N$ were dialyzed against the Tris-chloride buffer described below. PNGase F was purchased from New England BioLabs Inc. and digestions were performed according to the manufacturer's instructions.

\subsection{Buffer for hydrodynamic measurements on $\mathrm{N}$-terminal fragments of human laminin short arms}

All physicochemical characterization of laminin fragments was carried out in $20 \mathrm{mM}$ Tris/ $\mathrm{HCl}$ buffer supplemented with $150 \mathrm{mM} \mathrm{NaCl}$ and $5 \mathrm{mM} \mathrm{CaCl}_{2}$ : the ionic strength of this buffer, $\mathrm{pH} 8.5$ at $20^{\circ} \mathrm{C}$, is $0.17 \mathrm{M}$.

\subsection{Dynamic light scattering}

Dynamic light scattering profiles for $L M \alpha-1 \mathrm{~N}$ (wild-type and C49S mutant), LM $\alpha-5 \mathrm{~N}$ and LM $\beta-3 N$ in the Tris-chloride buffer were measured by means of the Zetasizer Nano S system (Malvern Instruments Ltd., Malvern, UK) equipped with a $4 \mathrm{~mW}$ laser $(\lambda=633 \mathrm{~nm})$ as described previously $[41,42]$. A stock solution of each laminin fragment was subjected to centrifugal filtration through a $0.1 \mu \mathrm{m}$ filter before dilution to yield a series of solutions with concentrations in the $0.2-3.5 \mathrm{~g} / \mathrm{L}$ range. These solutions were allowed to equilibrate for 4 min at $20{ }^{\circ} \mathrm{C}$ prior to DLS measurements at the same temperature. Multiple records of the DLS profile at each protein concentration were analyzed by means of the DTS software supplied by the manufacturer (Version 5.10.2, Malvern Instruments Ltd., Malvern, UK).

\subsection{Circular dichroism}

The secondary structures of all four variants (LM a-1 N - wild-type and C49S mutant, LM $\alpha-5 \mathrm{~N}$ and LM $\beta-3 \mathrm{~N}$ ) were examined by circular dichroism spectroscopy using a J-810 spectropolarimeter (Jasco, Japan). 50- $\mu \mathrm{L}$ aliquots of either protein solution $(1.1-1.3 \mathrm{~g} / \mathrm{L})$ or Tris-chloride buffer were loaded into a $0.1 \mathrm{~mm}$ demountable cell with open top (Starna Cells, Inc., Atascadero, CA). Each wavelength scan (including baseline) in 1-nm steps from 190 to $260 \mathrm{~nm}$ was measured in triplicate to obtain an average measurement as well as its standard deviation at each wavelength. Each wavelength point was measured for $16 \mathrm{~s}$ using a bandwidth of $1 \mathrm{~nm}$. The mean residue ellipticity was calculated per peptide bond as $[\theta]_{\mathrm{mrw}}=\theta /(C / n)$ where $\theta$ is the measured ellipticity in millidegrees, $C$ the molar concentration, / the optical path length (in $\mathrm{nm}$ ) of the 
cuvette, and $n$ the number of peptide bonds in the sample. Protein concentrations were determined spectrophotometrically at $280 \mathrm{~nm}$ on the basis of the average of three independent measurements from undiluted samples in a Nanodrop 2000c instrument (Thermo Scientific, Wilmington, DE). Molar extinction coefficients were calculated from the amino acid sequence of each fragment by means of the ExPASy ProtParam tool [13].

\subsection{Sedimentation velocity}

Sedimentation velocity experiments were performed in an Optima XL-I analytical ultracentrifuge (BeckmanCoulter, Palo Alto, CA) fitted with the An60-Ti rotor. Standard 12-mm double-sector cells were loaded with $380 \mu \mathrm{L}$ of laminin fragment solution $(0.5-0.6 \mathrm{~g} / \mathrm{L})$ and $400 \mu \mathrm{L}$ of Tris-chloride buffer in the sample and reference channels respectively. Solutions were subjected to centrifugation at $35,000 \mathrm{rpm}$ and $20{ }^{\circ} \mathrm{C}$, and solute distributions were recorded at 14-minute intervals by means of the absorption optical system set $280 \mathrm{~nm}$. Those distributions were analyzed by the SEDFIT program $[9,47]$ to obtain the weight-average sedimentation coefficient, $s_{20, b}$, which was then corrected to standard solvent conditions $\left(s_{20, w}\right)$ by means of the SEDNTERP program [30] and the partial specific volumes reported in Table 1: the SEDNTERP program was also used to calculate the buffer viscosity $(0.01022 \mathrm{P})$ required for that correction to standard conditions.

\subsection{Surface plasmon resonance}

The first set of SPR experiments were performed at $25^{\circ} \mathrm{C}$ using a Biacore 2000 instrument (GE Healthcare). The recombinant proteins hLM $\alpha-1 \mathrm{~N}$ wild-type and hLM $\alpha-1 \mathrm{~N}$ C49S, carrying a his-tag were coupled with the surface of a NTA-Chip [26]. For measurement of protein self-interaction, hLM a-1 N wild-type and $\alpha-1 \mathrm{~N}$ C49S respectively without tag were passed over the chip as soluble analytes in serial dilutions $(1-0.125 \mu \mathrm{M})$ in running buffer (10 mM Hepes pH 7.4, 150 mM NaCl, $50 \mu \mathrm{M}$ EDTA, 0.005\% P20). The experiments were performed at a constant flow rate of $30 \mu \mathrm{L} / \mathrm{min}$ with an association time of $300 \mathrm{~s}$ and a dissociation time of $500 \mathrm{~s}$. Fitting of the data and calculation of the $k_{a}, k_{d}$ and $K_{D}$ value were performed with the BIAevaluation 4.0 software using the 1:1 Langmuir binding model. An identically treated flow cell without coupled protein was used as a blank. Analogous experiments were performed with hLM $\alpha-1 \mathrm{~N}$ and its C49S mutant immobilized in random orientation on the traditional CM5 biosensor chip to examine the analyte-immobilized ligand interaction in buffer with the $50 \mu \mathrm{M}$ EDTA replaced by $2 \mathrm{mM} \mathrm{CaCl}_{2}$.

\section{Acknowledgments}

TRP was the recipient of a Canadian Institutes of Health Research postdoctoral fellowship and is currently supported by the Marie Skłodowska-Curie Fellowship. JS holds a Canada Research Chair in Structure Biology.

This investigation was supported in part by the Multiple Sclerosis Society of Canada and by the Canadian Institute of Health Research (RPA-109759).

\section{Appendix A. Supplementary data}

Supplementary data to this article can be found online at http://dx.doi.org/10.1016/j.matbio.2015.06. 005.

Received 1 June 2015; Received in revised form 25 June 2015;

Accepted 26 June 2015

Available online $x x x x$

Keywords:
Analytical ultracentrifugation;
CD spectroscopy;
Dynamic light scattering;
Extracellular matrix;
Laminin short arms;
Protein self-association;
Surface plasmon resonance

$\uparrow$ Both authors contributed equally.

\section{References}

[1] P. Antonsson, R.A. Kammerer, T. Schulthess, G. Hanisch, J. Engel, Stabilization of the alpha-helical coiled-coil domain in laminin by C-terminal disulfide bonds, J. Mol. Biol. 250 (1995) 74-79.

[2] M. Aumailley, The laminin family, Cell Adh. Migr. 7 (2013) 48-55.

[3] M. Aumailley, L. Bruckner-Tuderman, W.G. Carter, R. Deutzmann, D. Edgar, P. Ekblom, J.r. Engel, E. Engvall, E. Hohenester, J.C.R. Jones, H.K. Kleinman, M.P. Marinkovich, G.R. Martin, U. Mayer, G. Meneguzzi, J.H. Miner, K. Miyazaki, M. Patarroyo, M. Paulsson, V. Quaranta, J.R. Sanes, T. Sasaki, K. Sekiguchi, L.M. Sorokin, J.F. Talts, K. Tryggvason, J. Uitto, I. Virtanen, K. von der Mark, U.M. Wewer, Y. Yamada, P.D. Yurchenco, A simplified laminin nomenclature, Matrix Biol. 24 (2005) 326-332.

[4] K. Beck, T.W. Dixon, J. Engel, D.A. Parry, Ionic interactions in the coiled-coil domain of laminin determine the specificity of chain assembly, J. Mol. Biol. 231 (1993) 311-323.

[5] E.E. Brunbaugh, G.K. Ackers, Molecular sieve studies of interacting protein systems. 3. Measurement of solute 
partitioning bu direct ultraviolet scanning of gel columns, J. Biol. Chem. 243 (1968) 6315-6324.

[6] F. Carafoli, S.A. Hussain, E. Hohenester, Crystal structures of the network-forming short-arm tips of the laminin beta1 and gamma1 chains, PLoS ONE 7 (2012) e42473.

[7] H. Colognato, P.D. Yurchenco, Form and function: the laminin family of heterotrimers, Dev. Dyn. 218 (2000) 213-234.

[8] A.R. Cooper, M. Kurkinen, A. Taylor, B.L. Hogan, Studies on the biosynthesis of laminin by murine parietal endoderm cells, Eur. J. Biochem. 119 (1981) 189-197.

[9] J. Dam, P. Schuck, Calculating sedimentation coefficient distributions by direct modeling of sedimentation velocity concentration profiles, Methods Enzymol. 384 (2004) 185-212.

[10] M. Durbeej, Laminins, Cell Tissue Res. 339 (2010) 259-268.

[11] N. Fernandez-Fuentes, C.J. Madrid-Aliste, B.K. Rai, J.E. Fajardo, A. Fiser, M4T: a comparative protein structure modeling server, Nucleic Acids Res. 35 (2007) W363-W368.

[12] S. Fukazawa, E. Shinto, H. Tsuda, H. Ueno, A. Shikina, Y. Kajiwara, J. Yamamoto, K. Hase, Laminin beta3 expression as a prognostic factor and a predictive marker of chemoresistance in colorectal cancer, Jpn. J. Clin. Oncol. 45 (2015) 533-540.

[13] E. Gasteiger, C. Hoogland, A. Gattiker, S. Duvaud, M.R Wilkins, R.D. Appel, A. Bairoch, Protein identification and analysis tools on the ExPASy server, in: J.M. Walker (Ed.), The Proteomics Protocols Handbook, Humana Press 2005, pp. 571-607.

[14] G.A. Gilbert, Sedimentation and electrophoresis of interacting substances. I. Idealized boundary shape for a single substance aggregating reversibly, Proc. Roy. Soc. London A250 (1959) 377-388.

[15] S. Goldberg, T.L. Adair-Kirk, R.M. Senior, J.H. Miner, Maintenance of glomerular filtration barrier integrity requires laminin alpha5, J. Am. Soc. Nephrol. 21 (2010) 579-586.

[16] C. Heng, O. Lefebvre, A. Klein, M.M. Edwards, P. SimonAssmann, G. Orend, D. Bagnard, Functional role of laminin alpha1 chain during cerebellum development, Cell Adh. Migr. 5 (2011) 480-489.

[17] E. Hohenester, P.D. Yurchenco, Laminins in basement membrane assembly, Cell Adh. Migr. 7 (2013) 56-63.

[18] C.C. Huang, D.H. Hall, E.M. Hedgecock, G. Kao, V. Karantza, B.E. Vogel, H. Hutter, A.D. Chisholm, P.D. Yurchenco, W.G. Wadsworth, Laminin alpha subunits and their role in C. elegans development, Development 130 (2003) 3343-3358.

[19] I. Hunter, T. Schulthess, J. Engel, Laminin chain assembly by triple and double stranded coiled-coil structures, J. Biol. Chem. 267 (1992) 6006-6011.

[20] S.A. Hussain, F. Carafoli, E. Hohenester, Determinants of laminin polymerization revealed by the structure of the alpha5 chain amino-terminal region, EMBO Rep. 12 (2011) 276-282.

[21] N. Ichikawa-Tomikawa, J. Ogawa, V. Douet, Z. Xu, Y. Kamikubo, T. Sakurai, S. Kohsaka, H. Chiba, N. Hattori, Y. Yamada, E. Arikawa-Hirasawa, Laminin alpha1 is essential for mouse cerebellar development, Matrix Biol. 31 (2012) 17-28.

[22] S.E. Harding, P. Johnson, The concentration-dependence of macromolecular parameters, Biochem. J. 231 (1985) 5459-5555.

[23] G. Kao, C.C. Huang, E.M. Hedgecock, D.H. Hall, W.G. Wadsworth, The role of the laminin beta subunit in laminin heterotrimer assembly and basement membrane function and development in C. elegans, Dev. Biol. 290 (2006) 211-219.
[24] L.A. Kelley, M.J. Sternberg, Protein structure prediction on the Web: a case study using the Phyre server, Nat. Protoc. 4 (2009) 363-371.

[25] S.T. Kim, T.L. Adair-Kirk, R.M. Senior, J.H. Miner, Functional consequences of cell type-restricted expression of laminin alpha5 in mouse placental labyrinth and kidney glomerular capillaries, PLoS One 7 (2012) e41348.

[26] A.J. Kimple, R.E. Muller, D.P. Siderovski, F.S. Willard, A capture coupling method for the covalent immobilization of hexahistidine tagged proteins for surface plasmon resonance, Methods Mol. Biol. 627 (2010) 91-100.

[27] C. Kumagai, T. Kadowaki, Y. Kitagawa, Disulfide-bonding between Drosophila laminin beta and gamma chains is essential for alpha chain to form alpha betagamma trimer, FEBS Lett. 412 (1997) 211-216.

[28] M. Kurkinen, D.P. Barlow, J.R. Jenkins, B.L. Hogan, In vitro synthesis of laminin and entactin polypeptides, J. Biol. Chem. 258 (1983) 6543-6548.

[29] M.A. Larkin, G. Blackshields, N.P. Brown, R. Chenna, P.A. McGettigan, H. McWilliam, F. Valentin, I.M. Wallace, A. Wilm, R. Lopez, J.D. Thompson, T.J. Gibson, D.G. Higgins, Clustal W and Clustal $X$ version 2.0, Bioinformatics 23 (2007) 2947-2948.

[30] T.M. Laue, B.D. Shah, T.M. Ridgeway, S.L. Pelletier, Computer-aided interpretation of analytical sedimentation data for proteins, in: S.E. Harding, A.J. Rowe, J.C. Horton (Eds.), Analytical Ultracentrifugation in Biochemistry and Polymer Science, Royal Society of Chemistry, Cambridge, United Kingdom 1992, pp. 90-125.

[31] P.R. Macdonald, A. Lustig, M.O. Steinmetz, R.A. Kammerer, Laminin chain assembly is regulated by specific coiled-coil interactions, J. Struct. Biol. 170 (2010) 398-405.

[32] K.K. McKee, D. Harrison, S. Capizzi, P.D. Yurchenco, Role of laminin terminal globular domains in basement membrane assembly, J. Biol. Chem. 282 (2007) 21437-22147.

[33] J.E. Mellerio, R.A. Eady, D.J. Atherton, B.D. Lake, J.A. McGrath, E210K mutation in the gene encoding the beta3 chain of laminin-5 (LAMB3) is predictive of a phenotype of generalized atrophic benign epidermolysis bullosa, $\mathrm{Br}$. J. Dermatol. 139 (1998) 325-331.

[34] J.H. Miner, C. Li, Defective glomerulogenesis in the absence of laminin alpha5 demonstrates a developmental role for the kidney glomerular basement membrane, Dev. Biol. 217 (2000) 278-289.

[35] J.H. Miner, C. Li, J.L. Mudd, G. Go, A.E. Sutherland, Compositional and structural requirements for laminin and basement membranes during mouse embryo implantation and gastrulation, Development 131 (2004) 2247-2256.

[36] J.H. Miner, P.D. Yurchenco, Laminin functions in tissue morphogenesis, Annu. Rev. Cell Dev. Biol. 20 (2004) 255-284.

[37] N.M. Nguyen, D.G. Kelley, J.A. Schlueter, M.J. Meyer, R.M. Senior, J.H. Miner, Epithelial laminin alpha5 is necessary for distal epithelial cell maturation, VEGF production, and alveolization in the developing murine lung, Dev. Biol. 282 (2005) 111-125.

[38] L. Ning, H. Kurihara, S. de Vega, N. Ichikawa-Tomikawa, Z. Xu, R. Nonaka, S. Kazuno, Y. Yamada, J.H. Miner, E. Arikawa-Hirasawa, Laminin alpha1 regulates age-related mesangial cell proliferation and mesangial matrix accumulation through the TGF-beta pathway, Am. J. Pathol. 184 (2014) 1683-1694.

[39] D.J. O'Shannessy, M. Brigham-Burke, K.K. Soneson, P. Hensley, I. Brooks, Determination of rate and equilibrium binding constants for macromolecular interactions using 
surface plasmon resonance: use of nonlinear least squares analysis methods, Anal. Biochem. 212 (1993) 457-468.

[40] U. Odenthal, S. Haehn, P. Tunggal, B. Merkl, D. Schomburg, C. Frie, M. Paulsson, N. Smyth, Molecular analysis of laminin $\mathrm{N}$-terminal domains mediating self-interactions, J. Biol. Chem. 279 (2004) 44504-44512.

[41] T.R. Patel, C. Bernards, M. Meier, K. McEleney, D.J. Winzor, M. Koch, J. Stetefeld, Structural elucidation of full-length nidogen and the laminin-nidogen complex in solution, Matrix Biol. 33 (2014) 60-67.

[42] T.R. Patel, M. Meier, J. Li, G. Morris, A.J. Rowe, J. Stetefeld, T-shaped arrangement of the recombinant agrin G3-lgG Fc protein, Protein Sci. 20 (2011) 931-940.

[43] M. Pathania, E.V. Semina, M.K. Duncan, Lens extrusion from Laminin alpha 1 mutant zebrafish, Sci. World J. 2014 (2014) 524929.

[44] A. Purvis, E. Hohenester, Laminin network formation studied by reconstitution of ternary nodes in solution, J. Biol. Chem. 287 (2012) 44270-44277.

[45] P.B. Robbins, Q. Lin, J.B. Goodnough, H. Tian, X. Chen, P.A. Khavari, In vivo restoration of laminin 5 beta 3 expression and function in junctional epidermolysis bullosa, Proc. Natl. Acad. Sci. 98 (2001) 5193-5198.

[46] H. Schägger, Tricine-SDS-PAGE, Nat. Protoc. 1 (2006) 16-22.

[47] P. Schuck, Sedimentation analysis of noninteracting and self-associating solutes using numerical solutions to the Lamm equation, Biophys. J. 75 (1998) 1503-1512.

[48] D.J. Scott, P.R. Patel, D.M.T. Besong, J. Stetefeld, D.J. Winzor, Examination of the discrepancy between size estimates for ovalbumin from small-angle X-ray scattering and other techniques, J. Phys. Chem. B 115 (2011) 10725-10729.

[49] E. Seiradake, C.H. Coles, P.V. Perestenko, K. Harlos, R.A. Mcllhinney, A.R. Aricescu, E.Y. Jones, Structural basis for cell surface patterning through NetrinG-NGL interactions, EMBO J. 30 (2011) 4479-4488.

[50] E.V. Semina, D.V. Bosenko, N.C. Zinkevich, K.A. Soules, D.R. Hyde, T.S. Vihtelic, G.B. Willer, R.G. Gregg, B.A. Link, Mutations in laminin alpha 1 result in complex, lens-independent ocular phenotypes in zebrafish, Dev. Biol. 299 (2006) 63-77.
[51] J. Soding, A. Biegert, A.N. Lupas, The HHpred interactive server for protein homology detection and structure prediction, Nucleic Acids Res. 33 (2005) W244-W248.

[52] C. Spenle, P. Simon-Assmann, G. Orend, J.H. Miner, Laminin alpha5 guides tissue patterning and organogenesis, Cell Adh. Migr. 7 (2013) 90-100.

[53] D.J. Winzor, Reconciliation of zonal and frontal studies on concentration-dependent migration in gel filtration, Biochem. J. 101 (1966) 30C-31C.

[54] D.J. Winzor, Analytical exclusion chromatography, J. Biochem. Biophys. Methods 56 (2003) 15-32.

[56] M.A. Wolman, V.K. Sittaramane, J.J. Essner, H.J. Yost, A. Chandrasekhar, M.C. Halloran, Transient axonal glycoprotein-1 (TAG-1) and laminin-alpha1 regulate dynamic growth cone behaviors and initial axon direction in vivo, Neural Dev. 3 (2008) 6 .

[57] K. Xu, Z. Wu, N. Renier, A. Antipenko, D. Tzvetkova-Robev, Y. Xu, M. Minchenko, V. Nardi-Dei, K.R. Rajashankar, J. Himanen, M. Tessier-Lavigne, D.B. Nikolov, Neural migration. Structures of netrin-1 bound to two receptors provide insight into its axon guidance mechanism, Science 344 (2014) 1275-1279.

[58] P.D. Yurchenco, P.S. Amenta, B.L. Patton, Basement membrane assembly, stability and activities observed through a developmental lens, Matrix Biol. 22 (2004) 521-538.

[59] P.D. Yurchenco, Y. Quan, H. Colognato, T. Mathus, D. Harrison, Y. Yamada, J.J. O'Rear, The alpha chain of laminin-1 is independently secreted and drives secretion of its beta- and gamma-chain partners, Proc. Natl. Acad. Sci. U. S. A. 94 (1997) 10189-10194.

[60] N.S. Zinkevich, D.V. Bosenko, B.A. Link, E.V. Semina, Laminin alpha 1 gene is essential for normal lens development in zebrafish, BMC Dev. Biol. 6 (2006) 13.

[61] R Core Team, R: A language and environment for statistical computing, R Foundation for Statistical Computing, Vienna, Austria, 2015. URL http://www.R-project.org/. 